\title{
Conflicto y colaboración en la organización y gestión universitaria: vida cotidiana y cultura institucional
}

\author{
Dr. Fidel Molina-Luque \\ Universidad de Lleida \\ molina@geosoc.udl.cat
}

\section{Resumen}

La universidad se debate entre un tipo de organización mecanicista y un tipo de organización orgánica. La organización y gestión universitaria aparece en ocasiones muy burocratizada y en otras parece plantear una estructura algo más flexible, priorizando los objetivos generales de la propia universidad en relación con las responsabilidades dibujadas concretamente. Tomamos como estudio de caso una universidad española (la Universidad de Lleida) donde hemos podido realizar un trabajo de campo intensivo, a través de una metodología cualitativa que ha comportado diversas técnicas de investigación: observación participante, entrevistas en profundidad y grupos de discusión. La observación participante y las entrevistas informales se ban llevado a cabo en este último quinquenio (2011-2016) sobre la base de trabajos anteriores de entrevistas en profundidad (5) y grupos de debate (7 de discusión y 3 triangulares). El estilo de dirección se ha mostrado entre "participativo" y "decisivo (autoritario)", propio de una organización, entre mecanicista y orgánica, que, después de una época de madurez, se encuentra en un momento de cambio. Entre otras conclusiones, se destacan la necesidad de una reestructuración en algunas áreas del Personal de Administración y Servicios (PAS); la necesidad de mejora de la coordinación y comunicación entre áreas y servicios; la necesidad de disminuir la burocracia, clarificando los procedimientos pertinentes; también se plantea la profesionalización de direcciones de servicios o perfiles intermedios altos, así como la clarificación de la función directiva en la gestión entre el Personal Docente e Investigador (PDI) y el PAS, estando atentos a las posibles 
causas y condicionantes (estructurales) de las "desmotivaciones" que se expresan entre algunos miembros de la comunidad universitaria. Es precisamente en el apartado de la vida cotidiana donde el personal se encuentra entre las ventajas y los inconvenientes de una universidad relativamente pequeña: tensiones y conflictos que pueden gestionarse de manera alternativa y positiva y otros que pueden enquistarse más fácilmente.

Palabras clave conflicto, socialización, vida cotidiana, organizaciones, universidad.

\section{CONFlict AND COLlaboration IN UNIVERSity ORgANizATION AND} MANAGEMENT: DAILY LIFE AND INSTITUTIONAL CULTURE

Abstract: The university is between a type of mechanistic organization and a type of organic organization. Sometimes the university organization and management is bureaucratic, but sometimes it seems to propose a more flexible structure, prioritizing the general objectives of the university itself in relation to the concrete responsibilities. We took as a case study a Spanish university (the University of Lleida) where we were able to carry out an intensive fieldwork, through a qualitative methodology that has involved several research techniques: participant observation, in-depth interviews and discussion groups. The participant observation and the informal interviews have been carried out in this last five years (2011-2016) based on previous works (5 indepth interviews, 7 discussion groups and 3 triangular groups). The style of direction has been shown between "participatory" and "decisive (authoritarian)", typical of an organization, between mechanistic and organic, which is in a moment of change (after a period of maturity). Among other conclusions, the need for restructuring in some areas of Administration and Services Staff is highlighted; the need to improve coordination and communication between areas and services and the need to reduce bureaucracy, clarifying the relevant procedures. It also considers the professionalization of service leadership or high intermediate profiles, as well as the clarification of the managerial function in the management between the Teaching and Research Staff and the Administration and Services Staff, being attentive to the causes and structural conditions of the "demotivation" that are expressed among some members of the university community. It is in the section of daily life, where the staff is among the advantages and disadvantages of a relatively small university: conflicts that can be managed in an alternative and positive way and others conflicts that are not resolved.

Key words: conflict, socialization, daily life, organizations, university. 


\section{Introducción ${ }^{1}$}

La universidad es una organización que se debate entre la continuidad y el cambio, entre la tradición y la innovación, siendo esta última el motor más potente de su consolidación y desarrollo. La universidad, como organización, es un sistema abierto que tiene, en este sentido, un equilibrio dinámico entre lo interno y lo externo. Por un lado debe dar respuesta a rápidos cambios en el entorno actual $y$ de futuro en el que está inserida $y$, en este sentido, se valora sus posibilidades como sistema orgánico. Pero por otro lado se manifiestan elementos de un sistema mecanicista, sobre todo, en relación con su estructura interna y una inercia de siglos (pero también actual) de estructura burocrática y especialización en los cargos. En ocasiones, y aunque no se da precisamente una tendencia a una comunicación $^{2}$ ni a una autoridad jerárquica vertical, entre miembros del colectivo del Personal de Administración y Servicios - PAS — ( y, en menor medida, también del Personal Docente e Investigador - PDI-) se echa de menos elementos de un sistema mecanicista.

Por otra parte, el conflicto y la colaboración son dos caras de la misma moneda en, prácticamente, la totalidad de las organizaciones. Y ello, sobre todo, forma parte también de contextos dinámicos, de cambio. Precisamente porque colaboramos tenemos conflictos. Estos, de entrada, no son ni positivos ni negativos, depende de la gestión alternativa que se lleve a cabo y que pueda transformar los conflictos en oportunidades de mejora. La universidad como organización moderna no está exenta de estas situaciones colaborativas y conflictivas.

En todo caso, nos vamos a centrar en la situación estructural que, en ocasiones, va a provocar que aparezcan conflictos y/o que éstos no se resuelvan de la mejor manera posible (a veces hasta se puede ser eficaz, pero no eficiente). La universidad, en este sentido, se debate entre un tipo de organización mecanicista y un tipo de organización orgánica. La organización y gestión universitaria aparece en ocasiones muy burocratizada y en otras parece plantear una estructura

\footnotetext{
1 Este trabajo es fruto de tres etapas de gestión e investigación, a lo largo de dos décadas: a) en una primera etapa como Decano de una facultad (1998-2001 y 2001-2004), lo que permitió un primer acercamiento metodológico desde la observación participante conjugado con una formación teórica y práctica; $b$ ) en una segunda etapa como responsable de los tres primeros planes de formación del PAS (1998-2000, 2000-2002 y 2002-2004) y la realización de una investigación financiada por la Gerencia y las secciones sindicales sobre los recursos humanos en la universidad $(2007-2009)$ y c) en una tercera etapa de consolidación de la observación participante y reflexión, como Director del Centro de Formación Continua (2011-2015/16), responsable de PAS y PDI.

2 En la universidad, se potencia cada vez más la comunicación en red y las propias redes sociales (Factbook, Twister, etc.). La jerarquía de autoridad no es rígida, precisamente, sino que es flexible y existe permeabilidad entre los diferentes niveles jerárquicos. En ocasiones, esto último es tildado como un obstáculo para la gestión cotidiana (sobre todo por algunas personas del colectivo del PAS y también algún miembro del PDI cuando ocupa puestos de gestión).
} 
algo más flexible, priorizando los objetivos generales de la propia universidad en relación con las responsabilidades dibujadas concretamente. Es en este marco, en el que el PAS y el propio PDI desarrollan su labor de gestión y en el que se encuentran dificultades de liderar el cambio o, simplemente, facilitar la comunicación y la coordinación entre los diversos ámbitos, áreas de servicio y "recursos humanos"3 en general.

\section{Objetivos y metodología}

En esta línea, tres objetivos parecen pertinentes a nuestra investigación:

1. Analizar conceptualmente el tema de la tipología de organización en la universidad, con especial énfasis en las relaciones entre sus trabajadores (PDI y PAS)

2. Presentar una visión de conjunto de la cultura de la organización en la universidad, teniendo en cuenta la estructura organizativa y, sobre todo, la identidad profesional y la integración en la institución

3. Estudiar en profundidad la vida cotidiana en la universidad centrándonos en las posibilidades de la organización del trabajo, comunicación, coordinación y la relación con los compañeros del PAS y del PDI

La estructura básica de la temática del artículo consta de tres apartados de un contenido predominantemente empírico combinado con una revisión bibliográfica del tema en la que analizamos la contribución de diversos autores y diferentes aportaciones de estudios recientes y las conclusiones derivadas de todo ello.

En estos apartados se describen e interpretan desde una perspectiva cualitativa las relaciones y estructuras organizativas universitarias, haciendo hincapié en las relaciones cotidianas de sus trabajadores (sobre todo del PAS pero también del PDI). Finalmente hemos elaborado unas conclusiones que a partir de la síntesis de los resultados obtenidos pretenden aportar una serie de propuestas de mejora.

La metodología escogida se deriva de los objetivos propuestos en nuestra investigación, así, a través de la metodología cualitativa pretendemos desde una perspectiva más humanista, una interpretación de los hechos humanos, en una línea más bien vivencial-relacional, de percepción.

Lo cualitativo -como indica Alonso (1998)- es explícitamente praxiológico, incluso ideológico, ya que condiciona al propio investigador, posee un carácter crítico en una perspectiva abierta, concreta y dialéctica; su lógica situacional se

3 Presentamos entre comillas "recursos humanos", ya que en según que ambientes académicos, sobre todo, es una concepción no exenta de polémica. Quizá no es una expresión demasiado adecuada, ni cómoda ni feliz, pero es utilizada por la gran mayoría del propio PAS, gerencias, académicos, etc. 
basa en el aquí y ahora, sin huir de la incertidumbre. Lo cualitativo se mueve en el espacio de la intersubjetividad, la interpretación de la realidad social. Para construir la realidad intersubjetiva hay que recurrir a documentos y archivos, y/o crear situaciones discursivas que favorezcan esta situaciónł la observación participante, la entrevista abierta y los grupos de discusión, en nuestro caso.

Tomamos como estudio de caso una universidad española (la Universidad de Lleida) donde hemos podido realizar un trabajo de campo intensivo, a través - como acabamos de mencionar - de una metodología cualitativa que ha comportado diversas técnicas de investigación: observación participante, entrevistas en profundidad y grupos de discusión. La observación participante y las entrevistas informales se han llevado a cabo en este último cuatrienio (2011-2015) sobre la base de trabajos anteriores de entrevistas en profundidad (5) y grupos de debate ( 7 de discusión y 3 triangulares).

Las cinco entrevistas y los diez grupos de discusión (siete canónicos y tres triangulares) han sido seleccionados con los criterios pertinentes directamente relacionados con los objetivos de investigación, esto es, un liderazgo y/o una visión amplia e implicación directa desde diversos ámbitos de la vida laboral (académica) universitaria, en un entramado de representatividad discursiva (no estadística) en relación con la estructura organizativa universitaria (básicamente de gestión).

\section{La estructura organizativa y la cultura de la organización}

Las organizaciones pasan por una serie de etapas en el propio desarrollo de la misma organización, según las diferentes trayectorias históricas. La duración de las etapas puede ser más o menos extensa, pero la trayectoria sigue un cierto modelo determinado. En este sentido, y adaptando el esquema de Sánchez (1991: 131) y la formulación de Molina (2001 y 2008) podemos concretar una etapa o fase de inicio ${ }^{4}$, en la que los requerimientos básicos son de entendimiento, adaptación e impulso y que van a generar necesidades y demandas entorno al PAS. En una segunda fase de crecimiento, se da la creación y consolidación, por un lado, de la socialización profesional y por otro, de la identidad y la cultura de una organización que se desarrolla. En esta segunda fase, la necesidad más acentuada es la de "formación", la función prioritaria es la organización y destacan la "consulta" y la "dedicación" como estilos y actitudes fundamentales. La tercera fase de "madurez" tiene como función prioritaria la administración, un estilo de dirección

4 En nuestro estudio de caso, de la Universidad de Lleida se concreta con su creación (en su nueva etapa contemporánea) a partir del año 1992 (y no de su inicio histórico como Estudio General en 1300). 
participativo, siendo la actitud individual clave, la integración y la necesidad más acentuada, la información. La cuarta fase es la de "cambio", en la que la función prioritaria es la reestructuración, un estilo de dirección que debe bascular entre autoritario (decisivo) y participativo (por lo que comporta ese cambio importante en una institución ya madura), con una actitud individual de colaboración y una necesidad de "reciclaje".

De alguna manera estas son las cuatro fases "modélicas" en el desarrollo de una organización. Sin embargo, y en relación con nuestro estudio de caso de la UdL en plena crisis económica que ha afectado y afecta sobremanera a las universidades españolas en general, hemos incorporado una quinta fase de "consolidación y crisis", que bien puede entenderse como crisis de crecimiento y/o consolidación. Por ello, creemos que se redunda en los aspectos y requerimientos de la cuarta fase de "cambio", con un estilo de dirección "autoritario / participativo", ya que es necesario ante la crisis un liderazgo fuerte, pero combinado con una dirección participativa, ya que la organización está bien consolidada en un camino de madurez reconocido y reconocible. Vuelven a ser preponderantes los elementos de un sistema orgánico, pero o bien se dan, o bien se echan de menos elementos de un sistema mecanicista (reglas y procedimientos definidos por escrito, demanda de interacción vertical entre jefes y subordinados en el PAS). Hemos apuntado que la necesidad más acentuada es la de la "formación continua", ya que es la mejor opción ante cualquier situación de crisis y tendiendo hacia la consolidación. Incorporamos también aportaciones novedosas (además de estas últimas mencionadas) en relación con la actitud individual clave, que la identificamos en una mixtura, precisamente, de "colaboración y conflicto". Esta mezcolanza de colaboración y conflicto debe entenderse como una aportación en positivo, para poner sobre la mesa las dificultades en juego y que sea una oportunidad de mejora, como consecuencia de una colaboración enriquecedora, y superadora finalmente del conflicto.

\begin{tabular}{|l|l|l|l|l|l|}
\hline $\begin{array}{l}\text { Etapas } \\
\text { Udl }\end{array}$ & $\begin{array}{l}\text { Inicio } \\
(1992 \ldots)\end{array}$ & $\begin{array}{l}\text { Crecimiento } \\
(1997 \ldots)\end{array}$ & $\begin{array}{l}\text { Madurez } \\
(2003 / 04 \ldots)\end{array}$ & $\begin{array}{l}\text { Cambio } \\
(2007 / 08 \ldots)\end{array}$ & $\begin{array}{l}\text { Consolidación y crisis } \\
(2011 / 2016)\end{array}$ \\
\hline $\begin{array}{l}\text { Requerimientos } \\
\text { Función } \\
\text { Prioritaria }\end{array}$ & Impulso & Organización & Administración & Reestructuración & Consolidación \\
\hline $\begin{array}{l}\text { Estilo de } \\
\text { dirección válido }\end{array}$ & Autoritario & Consulta & Participativo & $\begin{array}{l}\text { Autoritario/ } \\
\text { Participativo }\end{array}$ & $\begin{array}{l}\text { Autoritario/ } \\
\text { Participativo }\end{array}$ \\
\hline $\begin{array}{l}\text { Actitud } \\
\text { individual clave }\end{array}$ & Adaptación & Dedicación & Integración & Colaboración & $\begin{array}{l}\text { Colaboración (y } \\
\text { Conflicto })\end{array}$ \\
\hline $\begin{array}{l}\text { Necesidad más } \\
\text { acentuada }\end{array}$ & Entendimiento & Formación & Información & “Reciclaje” & Formación Continua \\
\hline
\end{tabular}

Fuente: Elaboración propia, basado en el esquema de Sánchez (1991) y Molina (2008: 18) 
Este modelo sirve como guión de análisis o indicador de la evolución de la organización en concreto, ya que suele ser habitual que la trayectoria histórica de una organización influya en su estructura y su funcionamiento, en sus sistemas y en sus procesos. El hecho de llegar a la etapa de cambio o, incluso, de consolidación y crisis, puede ser analizado, desde nuestro punto de vista, como una cuestión meramente organizacional o como una cuestión que también tenga en cuenta los procesos individuales de integración, como es nuestro caso. Cuando se alcanzan actitudes de cooperación, las relaciones humanas se ven potenciadas, como una necesidad societaria que el sociólogo francés Crozier reivindica y plantea como positiva:

De la relación que se ha desarrollado a través de sus intercambios ha surgido un valor añadido. [...] Cuando se enseña así a la gente a valorar sus cualidades individuales en un trabajo en equipo, se constata que están verdaderamente contentos de ello. (Crozier, 1996: 151)

De hecho, entre los propios miembros del PAS se valora muy positivamente el hecho que el estilo de dirección sea participativo, pero parece que en ocasiones falte una dirección algo más firme (se debaten entre la realidad de un sistema orgánico y la querencia de un sistema algo más mecanicista). También se reconoce que la misma idiosincrasia de la institución universitaria no lo facilita, ya que los mismos profesores (PDI) que están en la gestión, están de manera temporal y tienen una presión, en ocasiones muy explícita, pero en otras ocasiones implícitamente; y también se ha de tener en cuenta las relaciones externas, con otras administraciones:

Se intenta consensuar mucho; el consenso es positivo [... Pero falta una visión más clara [...] Hay muchos obstáculos en el sistema público... A ver quien se atreve a dar un puñetazo en la mesa... Qué profesor que tiene un cargo y después vuelve a su departamento... También con el exterior, se ha de estar bien con las otras administraciones... Mandar debe ser muy difícil en la administración pública. (GD7)

Hemos de recordar, siguiendo el esquema mostrado, que el modelo de dirección participativo es el adecuado, pero si nos situamos ya en una etapa de crisis - como la que creemos que se está, 2015 - la dirección ha de combinar este estilo participativo con un estilo "autoritario" en el sentido de la "autoritas", con más implicación y firmeza a la hora de tomar las decisiones que le concierne.

En relación con la dirección y gestión de equipos, a veces se da una cierta disfuncionalidad de los jefes en relación con el resto del personal. En el caso de las universidades, y la UdL no es una excepción, esta situación es un punto débil en 
cuanto algunos directivos (PDI) no están del todo capacitados o no tienen todas las competencias para liderar el ámbito del cual se es responsable; en ocasiones no sabe escuchar a los técnicos o, simplemente, no ha tenido la formación suficiente que requiere su puesto de autoridad y liderazgo:

Depende del ámbito del que provenga, y del apoyo que tenga del administrativo; si tiene conocimientos, capacidades... La ley favorece que pase, queda al criterio del cargo, de la persona que asume la responsabilidad. (GT1)

Incluso se habla de la necesidad de profesionalizar las direcciones de los servicios o perfiles intermedios altos, al lado del cargo "político" (del académico), pero técnico que asegure esta cierta continuidad y estabilidad en las decisiones o en el desarrollo fundamental de la gestión:

El rector ha de hacer de rector, los vicerrectores han de hacer de vicerrectores, pero pienso que hay unos cargos intermedios que deberían estar mucho más profesionalizados [...] Profesionalizar desde arriba, pero que este "de arriba" también tuviera un poco las manos libres para poder hacer algunos fichajes externos y para ayudarlo a él. Que en ocasiones esto está mal visto dentro del estamento universitario, de traer gente de fuera hacia aquí dentro y cuando parece que hay gente que puedes promocionar $y$, bueno, si hay, pues muy bien de promocionarlos, pero a veces este agujero no lo puedes cubrir y se queda por cubrir muchas veces. (EP1)

Se reconoce que la estructura universitaria es muy compleja, jerárquica, combinando mandos más técnicos y más "políticos" (de académicos), que suelen ser miembros del PDI que necesitan más preparación para la gestión:

Es una estructura compleja, jerárquica [... Es necesaria una mayor preparación del PDI para gestionar. [...] Es necesario protocolizar la parte más técnica, no las líneas políticas (objetivos, orientaciones, criterios); tiene que haber una metodología establecida para los procedimientos de actuación. (GD5)

En ocasiones se pueden producir conflictos entre dos cargos (jefes) docentes o entre docentes i PAS... Se ha de clarificar las atribuciones de cada uno. Hay cargos que ejercen los docentes innecesariamente. En el caso de los Planes de Emergencia, no tendría que ser el decano el referente sino un PAS, por ejemplo. (GT3)

La imagen externa de la universidad ("de cara a fuera") parece que se valora como positiva, que va mejorando, pero que es insuficiente. En este sentido, se resalta que las acciones cotidianas suelen ser las que hacen que la imagen transcienda como positiva o no (de cara a los mimos estudiantes y a otras personas externas a la propia universidad): 
"Falta cultura corporativa, imagen de la UdL. Falta más por parte de los profesores que del PAS... Importa la implicación con la institución del día a día. [... ] A veces, los profesores tardan en firmar las actas de notas y eso impide la continuación de otros quehaceres, por ejemplo" (GD5)

Externamente (digo): "trabajo en la universidad". Pero internamente es algo diferente, "alegría frustrada"; es triste decirlo, tienes sueldo a final de mes, pero la cultura de la empresa es algo pobre,, desmotivación [... . A nivel individual, orgullosa,"es un lujo trabajar en la universidad. (GT1)

Hay una idea generalizada que, entre el PAS hay más cultura e identificación con la UdL que quizá entre el PDI, aunque en cualquier caso no es excesivamente alta esta identificación. Esto se ve sobre todo en la gestión, en el sentido de que los cargos políticos del PDI son temporales y los funcionarios permanecen. Ello comporta, en ocasiones, conflictos derivados de la resistencia al cambio, por ejemplo:

El funcionario permanece y el cargo político se desplaza. Se genera una cultura de empresa de base, alterada por el político que llega. Se genera resistencia: yo resisto en el lugar de trabajo, pase lo que pase. A veces es una cultura de estancamiento, de resistencia pasiva. (EP5)

También se repite la queja de que no se escuchan las propuestas que están presentadas sobre la base de la experiencia. Hay autonomía funcional, pero no se entra en el proceso de tomar decisiones. No se da la posibilidad de discutir los temas para que vayan mejor, pero en cambio se pide responsabilidad de "que todo salga bien". Realmente ello no solamente redunda en la propia dinámica cotidiana de la propia institución, sino que también está muy ligada a la imagen de la universidad.

En todo caso, la experiencia del PAS es fundamental y decisiva para que el PDI que asume un nuevo cargo pueda hacerlo con éxito (en la gran mayoría de ocasiones esto es así y la implicación es máxima y eficiente). El PAS suele estar más identificado con la universidad como estructura. De todas maneras, en ocasiones, se puede estar más identificado con el espacio que se ocupa y se reduce el espacio de ocupación, que, aunque también puede ocurrir entre el PAS, parece ser más frecuente entre el PDI:

Tendría que ser a la inversa, primero se es trabajador de la UdL, después de un departamento o un centro (facultad), etc. El profesorado le pasa más todavía, primero es de una cátedra o una área, después de un departamento... y finalmente de la UdL. (EP5) 
A pesar de todo, la motivación extrínseca e intrínseca es uno de los factores más importantes para la identidad profesional y la integración en la organización. La motivación es intrínseca cuando la propia persona, en su fuero interno, encuentra el aliciente per se en la misma actividad que desarrolla y de ser integrante de la organización. La motivación es extrínseca cuando necesita de "sanciones" positivas ("premios") y/o negativas ("castigos") para continuar con su labor de la mejor manera posible. No suelen estar necesariamente separadas, porque aunque aparentemente la motivación intrínseca no necesita de apoyo externo, finalmente aparece ligada a estímulos externos, económicos, de prestigio, de sentirse a gusto, etc. La motivación, pues, es también estructural (no depende meramente de actuaciones o elementos psicológicos, sino también sociológicos) y es uno de los factores clave en la cultura de la empresa, en la identidad profesional y en la integración en la universidad como organización.

Me siento integrado porque estoy motivado en el trabajo. ES necesario motivar a la gente, que se sienta a gusto, económicamente o no económicamente... reconocimiento. Otras mejoras laborales, sociales.

Otras personas no están motivadas [...] Dar más autonomía en el puesto de trabajo, tener iniciativa. En la formación de los cargos (jefes) debe estudiarse cómo motivar a los trabajadores. Tener una parcela de responsabilidad, también suele gustar, encontrarse a gusto. Es un revulsivo. También desde los PDI responsables; ha de cambiar la mentalidad del propio PDI. (GD6)

Una cultura empresarial determinada es una "imagen compartida" (Garmendia, 1990: 53) de los que componen la empresa y que por tanto ayuda a la cohesión y a la construcción de una identidad colectiva determinada. La imagen compartida suele ser también positiva entre los trabajadores de la UdL, sobre todo porque a nivel popular todavía la visión sobre la universidad, en el entorno local, es prestigiosa y reconocida, en ocasiones de manera aséptica, "por encima del bien y del mal", y eso impregna también a los trabajadores de la universidad, como integrantes de la misma; a pesar de que, internamente, pueda haber desmotivaciones por la dinámica del sistema:

Da prestigio ser funcionario... ser trabajador de la UdL. Aunque, a menudo, el PAS está poco motivado a sentirse suya la institución [...] De entrada te lo crees, se podrían hacer cosas, pero poco a poco el sistema te absorbe y no te lo crees tanto. Viste mucho que eres de la UdL, pero desde dentro se ve diferente. (GD7) 
La estima por la empresa creo que es alta [... $]$ Me gusta mucho pertenecer a la universidad y tengo un sentimiento muy positivo, pero pienso que en general la gente no lo tiene, no sabe... quizá también por eso es que te quemas de cosas de estas, vas perdiendo también un poco tu estima por la UdL.

[... Yo me siento integrado y estimo a la empresa y cuando alguno de fuera me dice alguna cosa me cabreo mucho, quiero decir que yo reacciono; entonces, esto es un vínculo afectivo que se va generando a lo largo de los años y... a ver, sí en términos generales, tu experiencia laboral y personal en esta empresa es positiva, evidentemente; yo pienso que acabas estimando y valorando mucho la empresa en la que estás, ¿no?. (GT2)

\section{Vida cotidiana: organización del trabajo, comuni- cación y relaciones interpersonales}

La vida cotidiana en la universidad es, finalmente, el núcleo fundamental para captar si la institución "funciona" o no. Es el termómetro que mide el día a día del desarrollo de la labor y de la propia organización. No únicamente es una cuestión de un clima laboral propicio basado en aparentes o reales buenas intenciones ("buen rollo") con la voluntad de que eso sea así, aunque también es bastante importante. Se ha de tener cuidado de aspectos que realmente facilitan o dificultan este clima laboral positivo; a veces son condiciones y/o condicionantes físicos (espacios, edificios, temperatura adecuada, etc.), otras de organización (distribución del tiempo de trabajo, colaboración, coordinación y comunicación) y también de la socialización en la propia institución (competitividad, cultura de la empresa, integración en la institución, identidad profesional).

Es todo en su conjunto, una complicada y compleja articulación de diversos elementos y factores aparentemente unos más "controlables" que otros, pero, insistimos que no son estrictamente personales e individuales, aunque el buen ánimo para ir a un trabajo que nos motive siempre ayuda a un buen clima laboral y a una relación Huacana y profesional más positiva y enriquecedora.

Se reconoce de manera prácticamente unánime y con una voluntad para que así sea destacado, el hecho de que el denominado "clima laboral", en general, entre el PAS se ha ido "relajando", en un sentido positivo y en otro sentido no tan positivo. Por un lado, hay un clima global menos tenso, de menos crispación que hace unos años. Pero, por otro lado, hay una cierta relajación en la organización o sobre todo, en la coordinación de las tareas que, dependiendo de los servicios y de las personas, provoca tensiones que son innecesarias. Algunos centran el debate 
en si haría falta que hubiera una normativa (procedimientos) más clara o ya lo está y lo que hace falta es aplicarla de manera más metódica y/o rigurosa; para no dejar tanto margen a la interpretación.

Hay percepciones concurrentes entorno a los procedimientos, considerando que la universidad se ha compartimentado mucho y que falta información y coordinación, o que se podría mejorar:

[...] el vicerrectorado correspondiente "manda" en lo suyo, no hay coordinación entre los otros vicerrectores $y$ otros servicios. Se quiere tener un punto de vista transversal, pero en la práctica es "estanca": hace falta coordinar las actuaciones, no que sean compartimentos estancos (vicerrectorados, negociados, etc.).(GD1)

Falta comunicación interna. [...] También hay demasiado volumen de trabajo, desorganización [...] trabajar por plazos te provoca mucha tensión. (GD 5)

Las cosas se han de explicar a la gente y se han de explicar bien, muchas veces el problema que hay es que no hay comunicación o se da una comunicación errónea, entonces no hay feedback. (GT2)

La vida cotidiana en la universidad, en lo que respecta a la organización del trabajo, la comunicación y la coordinación está también relacionada con la motivación, todo está imbricado de una manera u otra, y hace falta advertir, en términos generales, de los peligros que una falta de liderazgo, aunque sea parcial (en determinados ámbitos, áreas o servicios), pueden aguar el clima laboral de la universidad. Estas sensibilidades se aproximan más a unas consideraciones "mecanicistas" (búsqueda de estructura burocrática, jerarquía tradicional, identificación con el desarrollo interno de la organización más que de cara al exterior, organización como sistema cerrado) porque la organización universitaria aparece, de alguna manera, como un sistema orgánico:

Falta de liderazgo. Falta de objetivos claros. Hay incomunicación y desmotivación. [...] Me considero un absoluto desmotivado de la UdL, pero tengo mi círculo que me funciona, mi trabajo que me funciona, y sé que lo hago bien, porque al revés, no puede haber quejas de mi trabajo. (GD3)

En ocasiones el ambiente o el clima laboral se puede ver afectado negativamente por cuestiones más o menos colaterales como puede ser el hecho de los permisos o la realización de cursos que afectan indirecta o directamente en el volumen de faena de otros compañeros. Estas cuestiones se conectan con la demanda de que los directivos (jefes) asuman más claramente un papel de liderazgo y de poder de decisión (se está en el límite con el sistema orgánico de jerarquía flexible con énfasis en las relaciones humanas): 
Puede dar "mal ambiente" entre unos que hacen cursos en las horas de trabajo y otros que los hacen fuera del trabajo. Hace falta regularlo de forma más clara... si los mecanismos de responsabilidad dependen del carácter, eso lleva a esta situación [... No hay mecanismos que controlen al “jefe. (GT1)

Yo también tengo la sensación de que hay algunos servicios en los que los jefes no asumen todas las responsabilidades que les corresponderían y quizá de alguna manera intentan estar entre dos aguas, no? Bien ante la gerencia, bien ante sus trabajadores, sin complicarse demasiado en ninguno de los dos ámbitos; entonces esta falta de responsabilidad o de liderazgo o de implicación directa hace que quizá haya situaciones [... que no le haga llegar este hecho a la gerencia o situaciones de gerencia que no acaben de llegar al personal. (GT2).

La relación entre los compañeros del PAS y del PDI son generalmente buenas; de he hecho se repite una expresión bastante coloquial para ilustrarlo: se dice que hay "buen rollo". Después cuando se comienza a concretar el tema y a profundizar en según que tipo de reflexiones se encuentran matices a esta primera percepción general que, en cualquier caso, se mantiene hasta el final de las conversaciones.

Entre el propio colectivo del PAS se destaca que hay muy buena relación, independientemente de si es laboral o funcionario, de hecho cada vez hay menos homogeneidad en cada colectivo y hay labores o lugares de trabajo que no tienen la identificación tan clara como en otros momentos, de si es funcionario o laboral. Todo ello no obsta para reconocer que, como en otras organizaciones ( $y$ algunos destacan "en la Administración Pública"), haya envidias, "codazos", competitividad, quizá mobbing, etc. Pero parece que "no llega la sangre al río", y que se sienten compañeros.

La misma distribución por áreas, servicios, campus... hace que entre el colectivo del PAS, en su conjunto, no haya un conocimiento entre todos, pero también es "normal" en una institución que ha crecido en número de estudiantes (hasta ahora), de PDI y del propio PAS:

No nos conocemos entre el PAS, como mucho entre el Campus; a veces (nos conocemos) al realizar cursos. Antes se hacía la fiesta del PAS o la fiesta institucional, para interaccionar... En general no hay mal rollo. Ayuda también comer en el comedor (PDI y PAS juntos, de alguna manera). (GD6)

En términos generales, se insiste en que la relación entre el PAS es buena. Y ello se ha de destacar y se destaca. Ahora bien, sobre esta base se explican los diversos motivos que pueden hacer que, en ocasiones, las relaciones no sean idílicas; 
básicamente se debe a la comparación mezclada con una cierta desmotivación de algunos, previa a estas comparaciones o alentada a través de estas comparaciones (agravios comparativos):

La relación y el clima entre el PAS es bueno. Puntualmente se dan pequeñas fricciones [...] Falta valoración y eso lleva a la desmotivación. Depende de las personas; algunas personas hacen lo mínimo... También "aquí nunca pasa nada" y cobras igual. (GD4)

La relación del PAS con el PDI, desde el punto de vista del primer colectivo, es buena, correcta y cordial. Ello no obstante, es bastante común la idea de que las relaciones son jerarquizadas. Prácticamente la totalidad de los entrevistados han reconocido tener la percepción de que el PDI cree que está por encima del PAS, en términos generales. Eso no quita que pueda haber buena relación personal. Sin embargo, finalmente, se acaba admitiendo que dentro de la universidad, el PDI se cree, más o menos inconscientemente, algo más importante:

Hay también buen rollo, pero a veces el profesorado te hace ver que tú estás abajo [...]. Eres docente o no eres docente. El trato es correcto pero aunque sea inconscientemente... pero tienen esta superioridad. Es como un sistema de clases... eso está en el ambiente [...] Quizá entre el PAS también, pero no tan exagerado. (GD5)

La relación con el profesorado es buena, con excepciones. Hay quien no se siente compañero tuyo, otros no, se sienten compañeros. Antes la UdL, en el Estudio General, como era más pequeña, era más comunitaria. Pero siempre hay aquella línea entre el PAS y el profesorado... depende evidentemente de la persona, será más fina y más "gruesa", pero siempre se percibe que él es el docente y tú eres técnico. El docente se piensa que está por encima, el técnico se cree que está por debajo o se lo creen los dos. Hay una cultura muy diferenciada entre el PAS y el profesorado, y eso no es bueno para la empresa. Eso no quiere decir que no se pueda trabajar bien, que se hagan proyectos conjuntamente. Los profesores tienen el poder, y lo saben. El PAS está al servicio del profesorado, aunque depende de las personas [...] Raya más fina o no, pero casi siempre existe... (GD7)

Hay separación entre el profesorado y el PAS. En el Consejo de Gobierno el PAS participa y la asistencia suele ser plena, en cambio el profesorado, no. (GD7)

También se da entre el PDI la competición o la competitividad; no se pueden ver; envidias sanas y no tan sanas. Hay tensión, stress... No es solo entre el PDI... paga también el PAS, el mal rollo de algunos profes. (GD6) 
La proverbial distancia (cuando no desidia) que el profesorado marca con los "papeles" se traduce en una relación complicada en según qué momentos, porque puede haber pequeños conflictos en cuestiones más o menos burocráticas y que el PDI tenga la percepción que no se le facilitan las cosas como tendría que ser; o las dificultades, que también hemos mencionado con anterioridad, entre los cargos "políticos" docentes y técnicos del PAS:

Es una relación fluida, pero también hay problemas: a veces, el profesorado se queja con los servicios o el apoyo que se le facilita [...]; pero también es cierto que no siguen los procedimientos [...]. A veces tiene que ver con la falta de tiempo o que haya poco personal.

A veces se podría facilitar la labor del PAS, si los profesores leyesen la información, las circulares, etc. (GD2)

Hemos de conseguir más alumnos, más investigación, más transferencia, más... implicar más gente a hacerlo... más unión entre profesorado y PAS. El profesor ha de rellenar los papeles hasta el final [...] o lo puede hacer un PAS y el profesor puede dedicarse más a temas de pensar y de investigar de lo suyo, y tal y cual..."qué, tengo que hacer de esclavo del profesor?! No... cada uno su faena $[\ldots]$ hacer equipo". (EP3)

Desde el punto de vista de decir: "esto es un desastre porque el vicerrector...", el coordinador o el que sea que está por encima de él, no se entera?, tú dices... "Perdona, quién es el técnico? Tú le has puesto tres propuestas encima de la mesa para que pueda escoger?, le has explicado cada una de ellas? Tú has hecho tu labor de técnico, has analizado la situación, has hecho propuestas, las has debatido, y al final dices, mira únicamente tenemos estas dos opciones, y que el escoja políticamente". (EP2)

Se reconoce que en ocasiones, se da un exceso de información para el PDI y que el PAS ha de facilitar esta gestión de información:

A veces sí es cierto que hay un exceso de información para el profesorado y haría falta filtrarla; unos $y$ otros pueden estar desbordados.

Se ha de dar apoyo al profesorado, se tendría que dar más cosas "hechas", pero el PAS tampoco tiene tiempo; se ha de cubrir el día a día; faltan recursos.

A veces la dedicación del profesorado es global... gestión, investigación, docencia... es baja... y, a veces, no hay dedicación de implicación en las cuestiones más de gestión. (GD2)

El profesorado no quiere hacer papeles [...] no asume que los procedimientos son necesarios para el control y el buen funcionamiento. El PAS, en algún caso, no ha asumido que ha de cooperar para facilitarle el papel. El PDI quiere ser administrador pero no hacer papeles, pero genera papeles... (EP5) 
Una de les posibilidades de mejorar esta relación sería que el PDI fuera consciente de la necesidad de seguir los procedimientos y que facilitara el hecho "de rellenar papeles". Hace falta hacer un poco de pedagogía en este sentido. Se ha de formar al PDI en el procedimiento de la gestión que requiere, por ejemplo, saber porqué se han de hacer papeles (burocracia de funcionamiento); se ha de explicar el porqué, en una formación diaria. El mejor ejemplo es la valoración que se hace sobre la diferencia que se da entre el PDI que "ha pasado por la gestión" y el que no lo ha hecho. Ello no obstante, también hay quien considera que la gestión tendría que estar más profesionalizada, desde el PDI especializado en esta vertiente, o desde el mismo PAS:

No me gusta el modelo anglosajón porque es muy competitivo, pero realmente cuánto nos ahorraríamos si hubiera una carrera profesional como han hecho también en Holanda o Alemania, que un equipo rectoral que se dedique a la academia... que se dedique a la decencia y a la investigación... y que el equipo que lleva la gestión sean personas profesionales de carrera que ya sepan y no tengan que aprender desde el inicio todo [...]. Y, claro, nos ahorraríamos tiempo, dinero y continuidad;... cuando viene alguno nuevo, reinventamos la sopa de ajo... $y$ además te cansas de que te pregunten... y lo haces con gusto porque dices, ostras sí... porque todo el mundo tiene ganas de que vaya bien. (GD3)

De todas maneras, se destaca sobre manera la buena relación entre PAS y PDI, aunque, evidentemente, la relación individualizada entre afinidades personales, departamentos, etc. tiene mucha importancia en el día a día de la universidad, que tiene un clima "soportable" e incluso, bueno. Ahora bien, la culminación de un buen clima laboral pasa por un entramado complejo y transversal de relaciones personales, relaciones laborales, cultura de la empresa, estructura y organización institucional; todo ello contrastando percepciones diversas que posibiliten que el engranaje funcione, haciendo viable lo personal y lo institucional, en su justo término, con mesura y racionalidad:

Que tu clima laboral sea agradable, pero que eso lo hace... digamos por una parte tienes el clima provocado por tus compañeros y después otro de, diríamos, de la empresa, es decir, puedo estar bien con mis compañeros y el jefe directo, pero después puedo estar puteado porque me hacen venir un día por la tarde, o porque pido días personales y no me los dan, o porque quiero un curso de formación y no me lo dan... o sea es muy importante los dos lados, no? Por un lado, que tengas un ambiente laboral agradable y después que las condiciones laborales propias de la institución sean agradables, y lo que no te compensa uno, te lo ha de compensar el otro. (GT2) 
Hay tanta subjetividad en esto, también; tanta dificultad... que es muy complicado decir a esta persona le hacen mobbing y a esta no [...] Tendríamos que encontrar un mecanismo para que la gente se sintiera segura, pero no "pasara" a lo jeta... y también algún mecanismo para que el jeta pasara a trabajar. [...] Una de las funciones principales de los sindicatos no es ayudar al jeta, es que la institución funcione bien y el trabajador tenga los derechos reconocidos. (EP3)

\section{A modo de conclusión}

Entre otras conclusiones, se vislumbra que la universidad es, principalmente, un sistema orgánico, pero que la realidad cotidiana -con sus colaboraciones y conflictos- hace dudar de su entera adecuación al entorno, tanto interno como externo. Una parte importante de los entrevistados manifiestan la necesidad de algunos de los elementos de un sistema mecanicista, sobre todo en relación con liderazgos fuertes ("verticales") y con el establecimiento -y seguimiento- de unos procedimientos claros y bien definidos. De todas maneras, también se dan elementos de un sistema mecanicista y lo que se procura es que se tienda hacia un verdadero sistema orgánico: la necesidad de una reestructuración en algunas áreas del PAS; la necesidad de mejora de la coordinación y comunicación entre áreas y servicios y la necesidad de disminuir la burocracia, por ejemplo.

Entre esta mixtura, también se plantea la profesionalización de direcciones de servicios o perfiles intermedios altos, así como la clarificación de la función directiva en la gestión entre el PDI y el PAS y estar atentos a las posibles causas y condicionantes (estructurales) de las "desmotivaciones" que se expresan entre algunos miembros de la comunidad universitaria. Es precisamente en el apartado de la vida cotidiana donde el personal se encuentra entre las ventajas y los inconvenientes de una universidad relativamente pequeña: tensiones y conflictos que pueden gestionarse de manera alternativa y positiva y otros que pueden enquistarse más fácilmente (Molina, 2008).

En este sentido, podemos concretar, a modo de conclusión, una serie de propuestas de mejora de la institución (sobre todo, aunque no únicamente, desde la vertiente del PAS). Muchas de estas propuestas de mejora se derivan directamente de la superación de puntos débiles de la organización y otras son alternativas a diferentes situaciones actuales. También las relacionamos con elementos preponderantes de un sistema orgánico o mecanicista (o una cierta confluencia entre ambos, de alguna manera). De hecho, las propuestas, en su mayoría, sitúan a la universidad preponderantemente como un sistema orgánico: 
- La combinación de los dos estilos de dirección, entre autoritario (decisivo) y participativo (mezcolanda orgánico-mecanicista), puede ayudar en una organización como la universitaria a que las relaciones sean más armónicas y a que haya un enfoque correcto de la institución y de la relación laboral, para que haya unas verdaderas "actitudes amigas" para el trabajo (Calle, 2005). Esto último sobredimensiona un objetivo también clave en un sistema orgánico, como es las buenas relaciones interpersonales.

- La disminución de la "burocracia" puede ayudar a clarificar y concretar los procedimientos pertinentes para orientar las acciones (como confluencia mixta).

- Como sistema orgánico, la creación de equipos de trabajo para asegurar al máximo la coordinación y la comunicación entre áreas y servicios. Se podría plantear una "auditoría de comunicación" (Saló, 1997) para conocer con exactitud diversos aspectos que caracterizan los hábitos de comunicación y la cultura de la empresa. La auditoría de comunicación permite determinar el modelo de comunicación implantado en una organización, tanto en la comunicación interna como externa.

- Como sistema orgánico, se ha de profundizar en la socialización del PAS como trabajador de la universidad, para fortalecer l'ethos profesional del colectivo, como miembro de la comunidad universitaria. La autorrealización y la automotivación de los trabajadores, que se ha de tener en cuenta también en los planes de formación, pueden contribuir a alcanzar mejor calidad, eficacia, eficiencia y productividad en las organizaciones, en un clima laboral más gratificante (Balart, 1997). Promover, en este sentido y específicamente, la participación ("festiva", "como consumidor") del PAS en los actos públicos "académicos" por antonomasia como las inauguraciones de curso, entrega de orlas, etc.

- Como sistema orgánico, se ha de motivar a los trabajadores de la universidad a trabajar en equipo, para trabajar en red, tal y como se expresa en el decálogo de puntos fuertes sobre la Gestión del Conocimiento de Mayo y Lank (2007).

- Como sistema orgánico, se ha de estar atentos a las posibles causas y condicionantes de las "desmotivaciones" que se puedan dar. Se ha de reconocer explícitamente la labor bien hecha, y establecer fórums personalizados de debate e intercambios de opinión. Es recomendable fortalecer la figura del Ombudsman ("defensor del pueblo") universitario y ampliar 
las posibilidades de un verdadero servicio de Mediación y Resolución de Conflictos, que actúe no únicamente de forma reactiva, sino también -y sobre todo- de forma proactiva, consolidando un clima favorable que coadyuve a la mejora de la motivación de los trabajadores en particular (atención más personalizada). Incluir todas estas temáticas en la formación continua de los trabajadores: "aprender a aprender a trabajar", "organización del tiempo", "establecer prioridades", "Resolución de Conflictos" (Mediación, Gestión Alternativa de Conflictos), etc...

- Hemos de recordar que el conflicto se ha de abordar desde una nueva perspectiva más abierta y positiva, gestionándolo en beneficio de las propias personas y también de la institución. Incluso hay autores (Eisenhardt, Kahwajy y Bourgeois, 1997) que hablan de potenciar el conflicto y el desacuerdo entre los equipos de alta dirección para mejorar la organización.

- En ocasiones, como indica Ochoa (CIDEM, 2007), no se trata tanto de motivar como de no desmotivar ante los cambios de cultura organizativa, asegurando que se entienda bien la información y utilizarla.

- En este sentido, se ha de estar atentos a la motivación del personal, procurando que esta continúe creciendo y no decaiga. Se ha de impedir que se llegue a la "despedida interior" (El-Ghandouri, 2007) como consecuencia de un largo y complejo proceso de vivencias negativas, hasta el punto de que las diferencias entre las expectativas previas y la realidad se hace tan grande que genera una acumulación de dolor y decepción insoportable que lleva a la resignación laboral; incluso el autor habla de que esta infelicidad laboral puede llevar al trabajador a convertir el trabajo en una prisión.

- En las organizaciones universitarias (sobre todo públicas y sobre todo con el PAS) se debe establecer y/o repensar algún sistema claro y eficiente de sanciones positivas ("premios") y sanciones negativas para reforzar las acciones correctas y evitar las incorrectas. Siempre que sea posible se optará por la vía de las sanciones positivas que refuercen el trabajo bien hecho, y que no necesariamente han de ser económicas (o sí), sino que hay alternativas como los reconocimientos públicos, facilidades en la participación en otras actividades formativas, movilidad, etc.

- En las universidades podría y debiera haber una mayor flexibilidad en algunos horarios, según los puestos de trabajo y plantearse la posibilidad de trabajar por objetivos. Según los datos del "Euroíndice Laboral" de 
Adecco (2007) menos del 9\% de los trabajadores se beneficia de un horario flexible, mientras que la media europea es del $23 \%$; en este sentido, la diversidad y flexibilidad también es un valor en alza, ya que la creación de equipos diversos mejora los resultados de las compañías (Adecco, 2014). Es importante reflexionar y plantear propuestas para una nueva organización del tiempo: los horarios flexibles y los planes de conciliación (vida laboral-vida familiar) como aspectos muy relacionados con la igualdad y el cambio social.

- Para el desarrollo del buen clima laboral, es positivo facilitar encuentros más o menos informales entre los trabajadores, pero sin forzar la situación. Se puede continuar y ampliar actividades comunes como cenas de empresa, "aperitivos", etc. al estilo de los círculos de calidad japoneses, de recursos humanos. En cualquier caso, se ha de ser conscientes que más importante que esto es la buena coordinación y colaboración laboral cotidiana, ya que recordemos que los elementos estructurales de una institución y los valores que transmite influencian en el comportamiento de sus integrantes (Guillén, 2006).

- En la gestión "política" del PDI se ha de concienciar al PAS para que haga el trabajo técnico, planteando y facilitando alternativas para que el docente pueda decidir una opción entre otras, por ejemplo. Y se ha de concienciar al PDI de la importancia de escuchar y valorar la experiencia del PAS, evidentemente.

La mejora continua o kaizen ${ }^{5}$ no se basa tanto en los grandes cambios institucionales (que también se pueden dar) sino, sobre todo, en la mejora de las pequeñas cosas, incluso de manera imperceptible, dejando de lado lo que es superfluo. Hay quien se pregunta si la universidad, en general, está éticamente enferma; si la universidad está entre las organizaciones más sanas éticamente; si, por último, el nuevo modelo de universidad europea incidirá en la calidad ética de las universidades (Guillén, 2006). Esperemos que sí, ya que entre otros puntos más controvertidos, el Espacio Europeo de Educación Superior parece que apuesta por un modelo universitario que no sea de uniformidad, sino de armonización.

En todo caso, en esta tensión universitaria entre sistema orgánico o sistema mecanicista, o bien no se cumple totalmente la apuesta de Burns y Stalker, o bien el entorno en el que se mueve la universidad no es tan cambiante como parece. Por un lado, podemos pensar, a la luz de los resultados obtenidos, que se demandan elementos de un sistema mecanicista, sobre todo relacionados a

5 El método kaizen (mejora continua) ha sido creado por Masaka Imai -2007_, filósofo de empresa, entendiendo en positivo el cambio, que ha de ser aprovechado para mejorar (vid Molina, 2008). 
una jerarquía de autoridad más rígida y a unas reglas y procedimientos definidos claramente, aunque la situación del entorno sea de cambio y ello aconseje un sistema orgánico. Por otro lado, podemos pensar que el peso de la tradición de la universidad como buque insignia del conocimiento de una sociedad, influye en remodelar y/o "apaciguar" el supuesto entorno cambiante y en rápida transformación de nuestras sociedades contemporáneas, y hacer que precisamente lo apropiado en la organización universitaria, para su práctica administrativa, sea una gestión que se encuadre en un sistema mixto, entre mecanicista y orgánico. $\mathrm{O}$, finalmente, que sea considerado como un sistema orgánico con alma parcial y minoritariamente mecanicista.

\section{Bibliografía}

ADECCO (2007): Euroindice laboral Adecco (<http://www.adecco.es, 04/01/2008>).

ADECCO (2014): Gestión de la diversidad. El reto de las organizaciones 3.0 (<http://www.adecco.es,06/11/2014.http://www.adecco.es/_data/NotasPrensa/pdf/617.pdf 08/08/2015>).

Alonso, L.E. (1998). La mirada cualitativa en Sociología. Madrid, Fundamentos. BALART, J. (1997): "La inversión de la pirámide: la recuperación del equilibrio a través de la automotivación”, en Capital Humano, 106: 42-46.

Burns, T. y Stalker, G. M. (1961): The Management of Innovations. London, Tavistock.

Calle, R. (2005): La psicología del sosiego en el trabajo y la empresa. Barcelona, Integral.

CIDEM (2003): Guia de Gestión del Conocimiento. Barcelona, CIDEM (Centro de Innovación y Desarrollo Empresarial) (<http://www.cidem.com pdf, 03/01/2008>).

Claver, E.; Pertusa, E.M. y Molina, J.F. (2006): “Caracterización de las estructuras mecánica y orgánica a partir de las principales dimensiones estructurales", en Investigaciones Europeas de Dirección y Economía de la Empresa. Vol. $12(2): 187-204$.

Crozier, M. (1996): La crisis de la inteligencia. Madrid, MAP-BOE.

Eisenhardt, K.; Kahwajy, J. y Bourgeois, L. (1997): "Potencie el conflicto y el desacuerdo en lo equipos de alta dirección", en Harvard Deusto Business Review, 81: 12-26.

El-Ghandouri, L. (2007): El despido interior. Madrid, Alienta.

Garmendia, J. A. (1990): Desarrollo de la organización y cultura de la empresa. Madrid, Esic-Market. 
Guillén, M. (2006): Ética en las organizaciones. Construyendo confianza. Madrid, Pearson Prentice-Hall.

Lucas, A. (2013): Sociología de las organizaciones. Madrid, Fragua.

Mayo, A. y Lank, E. (2007): "10 preguntas para identificar los puntos fuertes en Gestión del Conocimiento" en Las organizaciones que aprenden. Madrid, Gestión 2000 (Revista Mundo Empresarial, Núm. Octubre, 2007: 38).

Molina, F. (2001): "La formación continua del Personal de Administración y Servicios de las universidades públicas", en La participación en las organizaciones: un desafío para el nuevo milenio. Zaragoza, Egido Editorial, p. 129-137.

Molina, F. (2008): Los recursos bumanos en la universidad. Lleida, Ediciones de la UdL.

Saló, N. (1997): “La auditoría de comunicación: esquema de realización", en Capital Humano, 106: 18-26.

Sánchez, M. (1991): La participación. Metodología y práctica. Madrid, Editorial popular.

Sine, W.D.; Mitsuhashi, H. y Kirsch, D.A. (2006): "Revisiting Burns and Stalker: formal structure and new venture performance in emerging economic sectors", en Academy of Managemente Journal. Vol. 49 (1): 121-132. 